\title{
Axiomatization of Some Basic and Modal Boolean Connexive Logics
}

\author{
Mateusz Klonowski
}

\begin{abstract}
Boolean connexive logic is an extension of Boolean logic that is closed under Modus Ponens and contains Aristotle's and Boethius' theses. According to these theses (i) a sentence cannot imply its negation and the negation of a sentence cannot imply the sentence; and (ii) if the antecedent implies the consequent, then the antecedent cannot imply the negation of the consequent and if the antecedent implies the negation of the consequent, then the antecedent cannot imply the consequent. Such a logic was first introduced by Jarmużek and Malinowski, by means of so-called relating semantics and tableau systems. Subsequently its modal extension was determined by means of the combination of possible-worlds semantics and relating semantics. In the following article we present axiomatic systems of some basic and modal Boolean connexive logics. Proofs of completeness will be carried out using canonical models defined with respect to maximal consistent sets.
\end{abstract}

Mathematics Subject Classification. Primary 03-02; Secondary 03B45, 03B60, 03C30, 03C50, 03C90, 03F99.

Keywords. Aristotle's laws, Axiomatic systems, Boethius' laws, Boolean connexive logics, Modalities, Relating logic, Relating semantics, Possibleworlds semantics.

\section{Introduction}

Boolean connexive logics are extensions of Boolean logic closed under Modus Ponens that contain connexive laws known as Aristotle's theses and Boethius' theses (cf. [11, p. 416], [13], [14, p. 371], [16]). Thus, any formula of the following schemata is valid in any Boolean connexive logic:

$$
\begin{aligned}
& \neg(A \rightarrow \neg A) \\
& \neg(\neg A \rightarrow A)
\end{aligned}
$$




$$
\begin{aligned}
& (A \rightarrow B) \rightarrow \neg(A \rightarrow \neg B) \\
& (A \rightarrow \neg B) \rightarrow \neg(A \rightarrow B) .
\end{aligned}
$$

Schemata (A1), (A2) are usually called Aristotle's laws (or Aristotle's theses) while (B1), (B2) are Boethius' laws (or Boethius' theses) (see [14, p. 371], [16], cf. [11, p. 416], [13, p. 406]). Logics of this kind were first introduced by Jarmużek and Malinowski in [8] by means of so-called relating semantics. ${ }^{1}$ Such a semantics enables one to consider in a straightforward way various relationships that may have an impact on the interpretation of complex propositions built by various connectives (cf. [4], [5, p. 54], [6,7]). Examples of such relationships might be temporal order, content dependence or causality.

In the case of Boolean connexive logics negation, conjunction and disjunction are two-valued extensional connectives. Implication is defined by the truth-condition for material implication and a condition stating the existence of a relationship (connexion) between the antecedent and the consequent. Only under some conditions introduced in [8] any formula instantiating the schema (A1), (A2), (B1) or (B2) is valid in relating semantics. Such conditions are independent and correspond to connexive laws, if we consider models based on relations that are closed under negation (see [8, pp. 438-439]).

Moreover, Kapsner's conditions of weakly and strongly connexive logics presented in [10] as well as the condition of properly connexive logic (the nonsymmetry of connexive implication) given by Jarmużek and Malinowski in [8] are satisfied.

Some further steps in the analysis of Boolean connexive logics were made in [9] where some modal systems were introduced (cf. [15]). The logics as before were determined semantically, this time by means of possible-worlds semantics combined with relating semantics. The proof-theory was, as in the case of Boolean connexive logics in [8], given by means of tableau systems.

On the basis of the least modal Boolean connexive logic neither $(\mathrm{K})$ nor the law of the duality of the modal operators is valid. Furthermore well-known relational properties of the accessibility relation are not enough to validate modal laws such as (T), (D), (B), (4) and (5). Two methods of making such laws valid were presented in [9]. In this article we are going to focus on the first one and only briefly consider the second one.

The main goal of our article is to axiomatize some of the logics considered in $[8,9]$. More specifically, we will consider axiomatic systems for the least Boolean connexive logic, its non-modal extension generated by models based on relations that are closed under negation and some modal extensions (generated by models that validate well-known modal schemata). The metalogical analysis will be carried out by adapting the methods applied in the studies of classical and modal logic respectively (cf. [1, pp. 45-64], [2, pp. 111-126], [3, pp. 105-116]). The proofs of completeness for all analysed systems will

\footnotetext{
${ }^{1}$ In [12] Boolean connexive logic was used in the analysis of Lewis Carroll's barbershop paradox (cf. [11, pp. 418-419]).
} 
be delivered by means of canonical models defined with respect to maximal consistent sets.

The structure of the paper is as follows: in Sect. 2, we introduce the languages and notations used in the article; in Sect. 3, we present the semantic analysis of the least Boolean connexive logic and some of its extensions, including modal ones; in Sect.4, we present an axiomatization of these logics and prove soundness and completeness for them.

\section{Preliminaries: Languages and Notations}

In the article we consider two languages. The first is the Boolean connexive language $\mathcal{L}$ that consists of propositional variables $p_{0}, q_{0}, r_{0} \ldots$; connectives: $\neg$ (negation), $\wedge$ (conjunction), $\vee$ (disjunction); $\rightarrow$ (implication) and brackets:), $\left(.^{2}\right.$ The second language, a modal Boolean connexive language $\mathcal{L}_{\square}$, is the modal extension of $\mathcal{L}$. Thus, it is the language $\mathcal{L}$ which additionally contains modal operators: $\square$ (necessity) and $\diamond$ (possibility).

The set of propositional variables is denoted by Var. The set of formulas of the language $\mathcal{L}$ (resp. $\mathcal{L}_{\square}$ ) is defined in the standard way and denoted by For (resp. For $\square$ ). ${ }^{3}$ We will omit the outermost brackets in formulas. Obviously For $\subset$ For $\square$. We use two abbreviations: $A \supset B:=\neg A \vee B$ and $A \equiv B:=$ $(\neg A \vee B) \wedge(\neg B \vee A)$.

\section{Semantics}

\subsection{Relating Semantics}

Let us start our semantic analysis by introducing relating semantics for the language $\mathcal{L}$ (cf. [5-7]). A model for $\mathcal{L}$ (based on a relation $R$ ) is an ordered pair $\langle v, R\rangle$ such that $v$ is a valuation of $\operatorname{Var}$, i.e. $v: \operatorname{Var} \longrightarrow\{1,0\}$, and $R$ is a binary relation between formulas, i.e. $R \subseteq$ For $\times$ For. ${ }^{4}$ The second element of model for $\mathcal{L}$ allows us to take under consideration various non-logical connexions. By means of it we can bind such propositions that refer to states of affairs that are related in some way.

The valuation $v$ (resp. the relation $R$ ) of a model $\mathfrak{M}$ for $\mathcal{L}$ is denoted by $v_{\mathfrak{M}}$ (resp. $R_{\mathfrak{M}}$ ). By $\mathbf{R}$ we shall denote the class of all binary relations on For. Let $\mathbf{Q} \subseteq \mathbf{R}$. The class of models based on $\mathbf{Q}$ is the class of models for $\mathcal{L}$ based on any $R$ from $\mathbf{Q}$. Such a class is denoted by MQ. The class of all models for $\mathcal{L}$ is $M R$.

\footnotetext{
${ }^{2}$ In some articles on relating semantics and its connexive applications the symbol $\rightarrow^{w}$ was used to denote implication (see $[8,12]$ ). Such a symbol was introduced in order to distinguish between relating and material implication denoted sometimes as $\rightarrow$ (cf. [7]).

${ }^{3} \mathrm{By} \mathrm{c}(A)$ we shall denote the complexity of a formula $A$ defined in the standard way, starting with 0 for propositional variables.

${ }^{4}$ We write $R(x, y)$, if $R \subseteq \Sigma \times \Sigma$ holds between elements $x, y \in \Sigma$ and $\sim R(x, y)$, if does not.
} 
A formula $A \in$ For is true in $\mathfrak{M} \in \mathbf{M R}$ (in symb.: $\mathfrak{M} \mid A ; \mathfrak{M} \not \neq A$, if false) iff for every $B, C \in$ For:

$$
\begin{array}{ll}
v_{\mathfrak{M}}(A)=1, & \text { if } A \in \operatorname{Var} \\
\mathfrak{M} \not B, & \text { if } A=\neg B \\
\mathfrak{M} \models B \text { and } \mathfrak{M} \models C, & \text { if } A=B \wedge C \\
\mathfrak{M} \models B \text { or } \mathfrak{M} \models C, & \text { if } A=B \vee C \\
{[\mathfrak{M} \not \models B \text { or } \mathfrak{M} \models C] \text { and } R_{\mathfrak{M}}(B, C),} & \text { if } A=B \rightarrow C .
\end{array}
$$

Let $\Sigma \subseteq$ For. We will write $\mathfrak{M} \models \Sigma$ instead of for every $A \in \Sigma, \mathfrak{M}=A$.

In the case of negation, conjunction and disjunction, we have the standard extensional interpretation. The truth-condition for implication says that either the antecedent is false or the consequent is true and also the constituent formulas must be related. Hence, the Boolean interpretation of implication is enriched by the non-Boolean requirement of a relationship between propositions.

Let us observe that the notion of truth might be also defined with respect to a relation from $\mathbf{R}$. A formula $A \in$ For is true with respect to $R \in \mathbf{R}$ (in symb.: $R \models A$ ) iff for every valuation $v: \operatorname{Var} \longrightarrow\{1,0\},\langle v, R\rangle \models A .^{5}$

The notions of valid formulas and semantic consequence are defined in the standard way. Let $\mathbf{Q} \subseteq \mathbf{R}$ and $\Sigma \cup\{A\} \subseteq$ For.

- $A$ is a valid formula in the class $\mathbf{M Q}$ (in symb.: $=_{\mathrm{MQ}} A$ ) iff for every $\mathfrak{M} \in \mathbf{M Q}, \mathfrak{M}={ }_{\mathrm{MQ}} A$

- $A$ is a semantic consequence of $\Sigma$ in the class $\mathbf{M Q}$ (in symb.: $\Sigma \models_{\mathrm{MQ}} A$ ) iff for every $\mathfrak{M} \in \mathbf{M Q}$, if $\mathfrak{M} \models \Sigma$ then $\mathfrak{M} \models A$.

The notion of valid formula might be also defined by means of the notion of truth with respect to relations from $\mathbf{R}$. The following fact holds (see [7]):

Proposition 3.1. Let $A \in$ For and $\boldsymbol{Q} \subseteq \boldsymbol{R}$. Then, $\models_{\mathrm{MQ}} A$ iff for every $R \in \boldsymbol{Q}$, $R \models A$.

We say that $A \in$ For is valid in the class $\mathbf{Q} \subseteq \mathbf{R}$ (symb.: $\models_{\mathbf{Q}_{\mathbf{Q}}} A$ ) iff for every $R \in \mathbf{Q}, R=A$.

In order to validate Aritotle's and Botheius' theses we consider the following relational conditions discussed in $[8,9]$ :

$$
\begin{gathered}
\forall_{A \in \text { For }} \sim R(A, \neg A) \\
\forall_{A \in \text { For }} \sim R(\neg A, A) \\
\forall_{A, B \in \text { For }}(R(A, B) \Rightarrow \sim R(A, \neg B))
\end{gathered}
$$

\footnotetext{
${ }^{5}$ We write $R \models(S)$, if for every formula $A$ instantiating the schema $(S)$ we have $R \models A$. For example, $R \models(\mathrm{A} 1)$ means that for every formula $B$ instantiating the schema (A1), i.e. of the form of $\neg(A \rightarrow \neg A), R \models B$.
} 


$$
\begin{aligned}
& \forall_{A, B \in \text { For }} R(A \rightarrow B, \neg(A \rightarrow \neg B)) \\
& \forall_{A, B \in \text { For }} R(A \rightarrow \neg B, \neg(A \rightarrow B)) .
\end{aligned}
$$

These conditions are independent (see [8, p. 440, theorem 7.1]).

A relation that satisfies (a1), (a2) (resp. (b0), (b1), (b2)) shall be called an Aristotelian (resp. Boethian) relation. By a connexive relation we mean a relation that is Aristotelian and Boethian.

Let us notice that by [8, p. 435, theorem 5.1]:

- if $R \in \mathbf{R}$ is an Aristotelian (resp. Boethian) relation, then $R \models(\mathrm{A} 1)$, $R \models(\mathrm{A} 2)$ (resp. $R \models(\mathrm{B} 1), R \models(\mathrm{B} 2))$.

However, the opposite implications do not hold, i.e. neither Aristotle's laws nor Boethius' laws induce Aristotelian and Boethian relation respectively (see [8, pp. 435-436]). This is not true, however, for relations closed under negation (see [8, p. 438, theorem 6.1]). By closure under negation we mean the following condition:

$$
\forall_{A, B \in \mathrm{For}}(R(A, B) \Rightarrow R(\neg A, \neg B)) .
$$

By $\mathbf{C}$ (resp. $\mathbf{C}\urcorner$ ) we denote the class of all connexive relations (resp. the class of all connexive relations closed under negations). In the article we would like to consider the least Boolean connexive logic BC, i.e. the logic determined by the class $\mathbf{C}$ and the Boolean connexive logic with the closure under negation $\mathrm{BC}\urcorner$, i.e. the logic determined by the class $\mathbf{C}\urcorner$.

\subsection{Combination of Relating and Possible-World Semantics}

Let us now discuss an interpretation of the modal Boolean connexive language $\mathcal{L}_{\square}$. For this purpose, we combine a possible-world semantics with a relating one.

A modal frame is an ordered pair $\langle W, Q\rangle$ such that $W$ is non-empty set of possible worlds and $Q \subseteq W \times W$ is an accessibility relation. By a relating frame we mean an ordered pair $\left\langle W,\left\{R_{w}\right\}_{w \in W}\right\rangle$ such that $W$ is non-empty set of possible worlds and $\left\{R_{w}\right\}_{w \in W}$ is a family of binary relations $R_{w} \subseteq$ For $\square \times$ For $\square$, where $w \in W$. Notice that a relating frame can be reduced to $\left\{R_{w}\right\}_{w \in W}=$ $\left\{R_{w} \subseteq\right.$ For $\times$ For $\left.: w \in W\right\}$, for a given $W$ (cf. [9, p. 220]). A combined frame is a triple $\left\langle W, Q,\left\{R_{w}\right\}_{w \in W}\right\rangle$ such that $\langle W, Q\rangle$ is a modal frame and $\left\{R_{w}\right\}_{w \in W}$ is a relating frame. As we can see, in a combined (resp. relating) frame for every possible world we have a binary relation that makes it possible to consider various non-logical relationships. By $\mathbf{F}_{\square}\left(\operatorname{resp} . \mathbf{F}_{\mathbf{r}}, \mathbf{F}\right)$ we shall denote the class of all modal (resp. relating, combined) frames. Let $\mathbf{E}_{1} \subseteq \mathbf{F}_{\square}, \mathbf{E}_{2} \subseteq \mathbf{F}_{\mathbf{r}}$. The class of combined frames based on $\mathbf{E}_{1}, \mathbf{E}_{2}$ is the class of all combined frames $\left\langle W, Q,\left\{R_{w}\right\}_{w \in W}\right\rangle$ such that $\langle W, Q\rangle \in \mathbf{E}_{1}$ and $\left\{R_{w}\right\}_{w \in W} \in \mathbf{E}_{2}$. Such a class is denoted by $\left[\mathbf{E}_{1}, \mathbf{E}_{2}\right]$. Thus $\mathbf{F}=\left[\mathbf{F}_{\square}, \mathbf{F}_{\mathbf{r}}\right]$.

A model for $\mathcal{L}_{\square}$ (based on a combined frame $\left\langle W, Q,\left\{R_{w}\right\}_{w \in W}\right\rangle$ ) is an quadruple $\left\langle W, Q,\left\{R_{w}\right\}_{w \in W}, v\right\rangle$ such that $\left\langle W, Q,\left\{R_{w}\right\}_{w \in W}\right\rangle$ is a combined frame and $v$ is a valuation of $\operatorname{Var}$ in possible worlds, i.e. $v: W \times \operatorname{Var} \longrightarrow\{1,0\}$.

A set of possible-worlds $W$ (resp. a relation $Q$, a valuation $v$ ) of a model $\mathfrak{M}$ for $\mathcal{L}_{\square}$ is denoted by $W_{\mathfrak{M}}$ (resp. $\left.Q_{\mathfrak{M}}, v_{\mathfrak{M}}\right)$. Let $\mathbf{E} \subseteq \mathbf{F}$. The class of models 
based on $\mathbf{E}$ is the class of all models for $\mathcal{L}_{\square}$ based on any frame from $\mathbf{E}$. Such a class of models is denoted by ME. The class of all models for $\mathcal{L}_{\square}$ is $\mathbf{M F}=\mathbf{M}\left[\mathbf{F}_{\square}, \mathbf{F}_{\mathbf{r}}\right]$.

Truth in a model for $\mathcal{L}_{\square}$ is defined in the standard way. A formula $A \in$ For $\square$ is true in a model $\mathfrak{M} \in \mathbf{M F}$, in a world $w \in W_{\mathfrak{M}}$ (in symb: $\mathfrak{M}, w \models A$; $\mathfrak{M}, w \not \models A$, if false) iff for every $B, C \in$ For $_{\square}$ :

$$
\begin{array}{ll}
v_{\mathfrak{M}}(w, A)=1, & \text { if } A \in \operatorname{Var} \\
\mathfrak{M}, w \not \models B, & \text { if } A=\neg B \\
\mathfrak{M}, w \models B \text { and } \mathfrak{M}, w \models C, & \text { if } A=B \wedge C \\
\mathfrak{M}, w \models B \text { or } \mathfrak{M}, w \models C, & \text { if } A=B \vee C \\
(\mathfrak{M}, w \not \models B \text { or } \mathfrak{M}, w \models C) \text { and } R_{w}(B, C), & \text { if } A=B \rightarrow C \\
\forall_{u \in W_{\mathfrak{M}}}\left(Q_{\mathfrak{M}}(w, u) \Rightarrow \mathfrak{M}, u \models B\right), & \text { if } A=\square B \\
\exists_{u \in W_{\mathfrak{M}}}\left(Q_{\mathfrak{M}}(w, u) \text { and } \mathfrak{M}, u \models B\right), & \text { if } A=\diamond B .
\end{array}
$$

Let $\Sigma \subseteq$ For $\square$. We will write $\mathfrak{M}, w \models \Sigma$ instead of for every $A \in \Sigma, \mathfrak{M}, w \models A$. A formula $A \in$ For $_{\square}$ is true in a model $\mathfrak{M}$ (in symb.: $\mathfrak{M} \models A$ ) iff for every $w \in W_{\mathfrak{M}}, \mathfrak{M}, w \models A$. And $A$ is false in $\mathfrak{M}$ (in symb.: $\mathfrak{M} \not \models A$ ) iff $A$ is not true in $\mathfrak{M}$.

As in the case of relating semantics and modal logic, we can define notion of true with respect to various structures. For instance, in [9, p. 220] the following notions were introduced:

- $A \in$ For $_{\square}$ is true with respect to a combined frame $\left\langle W, Q,\left\{R_{w}\right\}_{w \in W}\right\rangle \in \mathbf{F}$ (in symb.: $\left\langle W, Q,\left\{R_{w}\right\}_{w \in W}\right\rangle \models A$ ) iff for every valuation $v: W \times \operatorname{Var} \longrightarrow$ $\{0,1\},\left\langle W, Q,\left\{R_{w}\right\}_{w \in W}, v\right\rangle \models A$

- $A \in$ For $_{\square}$ is true with respect to a modal frame $\langle W, Q\rangle \in \mathbf{F}_{\square}$ (in symb.: $\langle W, Q\rangle \models A)$ iff for every relating frame $\left\{R_{w}\right\}_{w \in W},\left\langle W, Q,\left\{R_{w}\right\}_{w \in W}\right\rangle \models$ $A$

- $A \in$ For $_{\square}$ is true with respect to a relating frame $\left\{R_{w}\right\}_{w \in W} \in \mathbf{F}_{\mathbf{r}}$ (in symb.: $\left\{R_{w}\right\}_{w \in W} \models A$ ) iff for every accessibility relation $Q \subseteq W \times W$, $\left\langle W, Q,\left\{R_{w}\right\}_{w \in W}\right\rangle=A .^{6}$

As before, we define valid formulas and semantic consequence in the standard way. Let $\Sigma \cup\{A\} \subseteq$ For $\square$ and $\mathbf{E} \subseteq \mathbf{F}$.

- $A$ is a valid formula in the class $\mathbf{M E}$ (in symb.: $\models_{\mathbf{M E}} A$ ) iff for every $\mathfrak{M} \in \mathbf{M E}$, for every $w \in W_{\mathfrak{M}}, \mathfrak{M}, w \models_{\mathbf{M E}} A$

- $A$ is a semantic consequence of $\Sigma$ in the class $\mathbf{M E}$ (in symb.: $\Sigma \models_{\mathbf{M E}} A$ ) iff for every $\mathfrak{M} \in \mathbf{M E}$, for every $w \in W_{\mathfrak{M}}$, if $\mathfrak{M}, w \models \Sigma$ then $\mathfrak{M}, w \models A$.

Obviously, valid formulas might be also defined by means of the notion of truth with respect to classes of combined frames. We get the following fact:

Proposition 3.2. Let $A \in$ For $\square$ and $\boldsymbol{E}_{1} \subseteq \boldsymbol{F}_{\square}, \boldsymbol{E}_{2} \subseteq \boldsymbol{F}_{r}$. Then:

\footnotetext{
${ }^{6}$ We write $\mathfrak{F} \models(\mathrm{S})$, if $\mathfrak{F}$ is a frame (modal, relating or combined) and for every $A \in$ For $\square$ instantiating the schema ( $\mathrm{S}), \mathfrak{F} \models A$.
} 
1. $\models_{\boldsymbol{M}\left[\boldsymbol{E}_{1}, \boldsymbol{E}_{2}\right]}$ A iff for every $\mathfrak{F} \in\left[\boldsymbol{E}_{1}, \boldsymbol{E}_{2}\right], \mathfrak{F} \models A$

2. $\models_{M\left[\boldsymbol{E}_{1}, \boldsymbol{F}_{\boldsymbol{r}}\right]}$ A iff for every $\mathfrak{F} \in \boldsymbol{E}_{1}, \mathfrak{F} \models A$

3. $\models_{M\left[\boldsymbol{F}_{\square}, \boldsymbol{E}_{2}\right]} A$ iff for every $\mathfrak{F} \in \boldsymbol{E}_{2}, \mathfrak{F} \models A$.

Proof. By definitions of valid formulas and formulas true with respect to a given structure.

We say that $A \in$ For $\square$ is valid in a class $\mathbf{E} \subseteq \mathbf{F}$ (in symb.: $\models_{\mathbf{E}} A$ ) in the case for every $\mathfrak{F} \in \mathbf{E}, \mathfrak{F}=A$.

Let $\left\{R_{w}\right\}_{w \in W} \in \mathbf{F}_{\mathbf{r}}$ and $w \in W$. We have the following modal counterparts of relational conditions discussed in the previous Sect. (see [9, pp. 221]):

$$
\begin{gathered}
\forall_{A \in \text { For }_{\square}} \sim R_{w}(A, \neg A) \\
\forall_{A \in \text { For }_{\square}} \sim R_{w}(\neg A, A) \\
\forall_{A, B \in \text { For }_{\square}}\left(R_{w}(A, B) \Rightarrow \sim R_{w}(A, \neg B)\right) \\
\forall_{A, B \in \text { For }_{\square}} R_{w}(A \rightarrow B, \neg(A \rightarrow \neg B)) \\
\forall_{A, B \in \text { For }_{\square}} R_{w}(A \rightarrow \neg B, \neg(A \rightarrow B)) .
\end{gathered}
$$

As before, by an Aristotelian (resp. Boethian) frame we mean a relating frame $\left\{R_{w}\right\}_{w \in W}$ such that for every $w \in W, R_{w}$ satisfies (a1 ${ }^{\square}$ ) and (a $2^{\square}$ ) (resp. (b0 $\left.0^{\square}\right),\left(\mathrm{b} 1^{\square}\right),\left(\mathrm{b} 2^{\square}\right)$ ), i.e. the family of Aristotelian (resp. Boethian) relations indexed by possible worlds. A connexive frame is a frame that is both Aristotelian and Boethian. By [9, p. 222, theorem 4.2]:

- if $\mathfrak{F} \in \mathbf{F}_{\mathbf{r}}$ is Aristotelian (resp. Boethian) frame, then $\mathfrak{F} \models(\mathrm{A} 1), \mathfrak{F} \models(\mathrm{A} 2)$ (resp. $\mathfrak{F} \models(\mathrm{B} 1), \mathfrak{F} \models(\mathrm{B} 2))$.

The opposite implications do not hold, but the proper closure under negation again makes it possible to get equivalence (see [9, pp. 221-222, theorem 4.1]).

By $\mathbf{C}_{\square}$ we denote the class of all combined frames based on the class of all modal frames and the class of all connexive frames for $\mathcal{L}_{\square}$. The least modal Boolean connexive logic $\mathrm{BC}_{\square}$ is the logic determined by the class $\mathbf{C}_{\square}$.

Since we want to deal with the modal version of Boolean connexive logics, the question naturally arises of which modal formulas are valid in such logics. Let us consider the following well-known modal schemata:

$$
\begin{gathered}
\diamond A \rightarrow \neg \square \neg A \\
\neg \square \neg A \rightarrow \diamond A \\
\square(A \rightarrow B) \rightarrow(\square A \rightarrow \square B) \\
\square A \rightarrow A \\
\square A \rightarrow \diamond A
\end{gathered}
$$




$$
\begin{gathered}
A \rightarrow \square \diamond A \\
\square A \rightarrow \square \square A \\
\diamond A \rightarrow \square \diamond A .
\end{gathered}
$$

As we know, formulas instantiating the schemata $\left(\mathrm{D}_{1}\right),\left(\mathrm{D}_{2}\right)$ and $(\mathrm{K})$ are elements of any normal modal logic, while formulas instantiating the schema (T) are elements of normal modal logics defined by reflexive modal frames, formulas instantiating the schema (D) are are elements of normal modal logics defined by serial modal frames, and so on. ${ }^{7}$

None of the above schemata is a schema of valid formulas in logic $\mathrm{BC}_{\square}$. And even if we consider some extensions of $\mathrm{BC}_{\square}$ defined by means of proper modal frames (for a given modal schema) the considered laws still will not be valid (see [9, pp. 223-224, claim 5.1]). But, it appears that $\mathrm{BC}_{\square}$ is closed under the Necessitation Rule. We have that if $A \in \mathrm{BC}_{\square}$, then $\square A \in \mathrm{BC}_{\square}$ (see [9, pp. 223-224, claim 5.1 (a)]). And obviously $\mathrm{BC}_{\square}$ contains formulas of the following schemata:

$$
\begin{gathered}
\diamond A \equiv \neg \square \neg A \\
\square(A \supset B) \supset(\square A \supset \square B) .
\end{gathered}
$$

In [9] two ways were presented in which we can validate the modal laws. The first one assumes some restrictions on modal and relating frames. We consider them below. The second way is similar to the first one, but it refers to a certain mapping called a demodalization. ${ }^{8}$ Such a function enables one to simplify constraints that are put on relating frames within the first approach. In this article we focus on the first way.

Let $\left\langle W, Q,\left\{R_{w}\right\}_{w \in W}\right\rangle \in \mathbf{F}$ and $w \in W$. In order to validate (K) the following conditions are introduced:

$$
\begin{gathered}
\forall_{A, B \in \text { For } \square} R_{w}(\square(A \rightarrow B), \square A \rightarrow \square B) \\
\forall_{A, B \in \text { For } \square}\left(\forall_{u \in W}\left(Q(w, u) \Rightarrow R_{u}(A, B)\right) \Rightarrow R_{w}(\square A, \square B)\right) .
\end{gathered}
$$

We say that a combined frame $\left\langle W, Q,\left\{R_{w}\right\}_{w \in W}\right\rangle$ satisfies (k1), (k2) iff for every $w \in W, R_{w}$ satisfies (k1) and for every $w \in W, Q$ and $R_{w}$ satisfies (k2). By [9, pp. 224-225, claim 5.2]:

- if $\mathfrak{F} \in \mathbf{F}$ satisfies $(\mathrm{k} 1),(\mathrm{k} 2)$, then $\mathfrak{F}=(\mathrm{K})$.

\footnotetext{
${ }^{7}$ As usual we assume that $Q \subseteq W \times W$ is: reflexive iff $\forall_{w \in W} Q(w, w)$; serial iff $\forall_{w \in W} \exists_{u \in W}$ $Q(w, u)$; symmetric iff $\forall_{w, u \in W}(Q(w, u) \Rightarrow Q(w, u))$; transitive iff $\forall_{w, u, t \in W}((Q(w, u)$ and $Q(u, t)) \Rightarrow Q(w, t))$; Euclidean iff $\forall_{w, u, t \in W}((Q(w, u)$ and $Q(w, t)) \Rightarrow Q(u, t))$. We say that a modal frame is $x y z$ iff its accessibility relation is $x y z$.

${ }^{8}$ The notion of demodalization was also introduced in [6] in order to consider a deontic relationship between propositions. Such a relationship does not depend on the modal status of propositions, but only their content. Notice that the demodalization of the kind considered in [9] is a simple eraser transformation defined in [1, pp. 22-23, exercise 1.27].
} 
The opposite implication does not hold. For instance, consider the following combined frame: $\left\langle W, Q,\left\{R_{w}\right\}_{w \in W}\right\rangle$ such that $W:=\{\alpha\}, Q:=\{\langle\alpha, \alpha\rangle\}$ and $R_{\alpha}:=\left\{\left\langle p_{0} \vee \neg p_{0}, p_{0} \wedge \neg p_{0}\right\rangle\right\} \cup\{\langle\square(A \rightarrow B), \square A \rightarrow \square B\rangle: A, B \in$ For $\square\} \cup$ $\left\{\langle\square A, \square B\rangle: A, B \in\right.$ For $\square$ and either $A \neq p_{0} \vee \neg p_{0}$ or $\left.B \neq p_{0} \wedge \neg p_{0}\right\}$ (cf. [9]).

Let $\left\{R_{w}\right\}_{w \in W} \in \mathbf{F}_{\mathbf{r}}$ and $w \in W$. In order to validate other modal laws we consider the following conditions:

$$
\begin{gathered}
\forall_{A \in \mathrm{For}_{\square}} R_{w}(\diamond A, \neg \square \neg A) \\
\forall_{A \in \mathrm{For}_{\square}} R_{w}(\neg \square \neg A, \diamond A) \\
\forall_{A \in \mathrm{For} \square} R_{w}(\square A, A) \\
\forall_{A \in \text { For } \square} R_{w}(\square A, \diamond A) \\
\forall_{A \in \text { For } \square} R_{w}(A, \square \diamond A) \\
\forall_{A \in \text { For } \square} R_{w}(\square A, \square \square A) \\
\forall_{A \in \text { For } \square} R_{w}(\diamond A, \square \diamond A) .
\end{gathered}
$$

We say that a relating frame $\left\{R_{w}\right\}_{w \in W}$ satisfies (xyz) iff for every $w \in W$, $R_{w}$ satisfies (xyz). By [9, pp. 225-226, claim 5.3] we have that:

- $\mathfrak{F} \in \mathbf{F}_{\mathbf{r}}$ satisfies (d1) iff $\mathfrak{F}=\left(\mathrm{D}_{1}\right)$

- $\mathfrak{F} \in \mathbf{F}_{\mathbf{r}}$ satisfies (d2) iff $\mathfrak{F}=\left(\mathrm{D}_{2}\right)$

- $\mathfrak{F} \in \mathbf{F}$ is based on a reflexive modal frame and a relating frame that satisfies (t) iff $\mathfrak{F}=(\mathrm{T})$

- $\mathfrak{F} \in \mathbf{F}$ is based on a serial modal frame and a relating frame that satisfies (d) iff $\mathfrak{F} \models(D)$

- $\mathfrak{F} \in \mathbf{F}$ is based on a symmetric modal frame and a relating frame that satisfies (b) iff $\mathfrak{F}=(\mathrm{B})$

- $\mathfrak{F} \in \mathbf{F}$ is based on a transitive modal frame and a relating frame that satisfies (iv) iff $\mathfrak{F} \models(4)$

- $\mathfrak{F} \in \mathbf{F}$ is based on an Euclidean modal frame and a relating frame that satisfies (v) iff $\mathfrak{F}=(5)$.

Taking under consideration the conditions above we introduce the following subclasses of $\mathbf{C}_{\square}$ :

- $\mathbf{C}_{\square} \mathbf{D}_{\mathbf{1}}$ is the sub-class of $\mathbf{C}_{\square}$ of combined frames based on the class of all modal frames and the class of relating frames such that (d1) is satisfied

- $\mathbf{C}_{\square} \mathbf{D}_{\mathbf{2}}$ is the sub-class of $\mathbf{C}_{\square}$ of combined frames based on on the class of all modal frames and the class of relating frames that satisfies (d2)

- $\mathbf{C}_{\square} \mathbf{K}$ the sub-class of $\mathbf{C}_{\square}$ of combined frames that satisfies (k1), (k2) 
- $\mathbf{C}_{\square} \mathbf{T}$ is the sub-class of $\mathbf{C}_{\square}$ of combined frames based on reflexive modal frames and relating frames that satisfies $(\mathrm{t})$

- $\mathbf{C}_{\square} \mathbf{D}$ is the sub-class of $\mathbf{C}_{\square}$ of combined frames based on serial modal frames and relating frames that satisfies (d)

- $\mathbf{C}_{\square} \mathbf{B}$ is the sub-class of $\mathbf{C}_{\square}$ of combined frames based on symmetric modal frames and relating frames that satisfies (b)

- $\mathrm{C}_{\square} \mathbf{4}$ is the sub-class of $\mathrm{C}_{\square}$ of combined frames based on transitive modal frames and relating frames that satisfies (iv)

- $\mathbf{C}_{\square} \mathbf{5}$ is the sub-class of $\mathbf{C}_{\square}$ of combined frames based on Euclidean modal frames and relating frames that satisfies (v).

By a class $\mathbf{C}_{\square} \mathbf{X}_{\mathbf{1}} \ldots \mathbf{X}_{\mathbf{n}}$ we mean the intersection $\mathbf{C}_{\square} \mathbf{X}_{\mathbf{1}} \cap \ldots \cap \mathbf{C}_{\square} \mathbf{X}_{\mathbf{n}}$. Hence, for instance, $\mathbf{C}_{\square} \mathbf{K T} \mathbf{5}$ is the sub-class of $\mathbf{C}_{\square}$ of combined frames that satisfies (k1), (k2) and are based on reflexive, Euclidean modal frames and relating frames that satisfies $(\mathrm{t}),(\mathrm{v})$. The logic determined by the class of frames $\mathbf{C}_{\square} \mathbf{X}_{\mathbf{1}} \ldots \mathbf{X}_{\mathbf{n}}$ shall be denoted by $\mathrm{BC}_{\square} \mathrm{X}_{1} \ldots \mathrm{X}_{\mathrm{n}}$.

\section{Axiomatization}

\subsection{Axiomatic Systems}

Let us now present the axiomatization of the logics under consideration here. By PL (resp. PL $\square$ ) we shall denote the set of all formulas which are instances of classical tautologies in the language $\mathcal{L}$ (resp. $\mathcal{L}_{\square}$ ), where $\neg, \wedge, \vee$ are the only classical connectives.

By an axiomatic system we mean any set $\Sigma \subseteq$ For such that $\mathrm{PL} \subseteq \Sigma$; $\Sigma$ contains $\mathcal{L}$ 's schemata (A1), (A2), (B1), (B2) and the following $\mathcal{L}$ 's schema: ${ }^{9}$

$$
(A \rightarrow B) \supset(A \supset B),
$$

$\Sigma$ is closed under a slight modification of Disjunctive Syllogism:

$$
\text { if } A, A \supset B \in \Sigma \text { then } B \in \Sigma \text {. }
$$

By (Imp) and (DS) any axiomatic system $\Sigma$ is closed under Modus Ponens, i.e.:

$$
\text { if } A, A \rightarrow B \in \Sigma \text { then } B \in \Sigma \text {. }
$$

The intended axiomatic system for BC is the least axiomatic system and shall be denoted by $\mathrm{S}$. The intended axiomatic system for $\mathrm{BC}\urcorner$ is the system $\mathrm{S}\urcorner$ that is the least axiomatic system that contains the following $\mathcal{L}$ 's schemata:

$$
\begin{gathered}
(A \rightarrow B) \supset((\neg A \rightarrow \neg B) \vee(\neg A \wedge B)) \\
(A \rightarrow B) \supset(\neg \neg A \rightarrow \neg \neg B) .
\end{gathered}
$$

By a modal axiomatic system we mean any set $\Sigma \subseteq$ For $\square$ such that $\mathrm{PL}_{\square} \subseteq \Sigma$; $\Sigma$ contains the $\mathcal{L}_{\square}$ 's schemata (Imp), (A1), (A2), (B1), (B2), (Dual ${ }^{\equiv}$ ) and

\footnotetext{
${ }^{9}$ We say that $\Sigma$ contains $\mathcal{L}$ 's (resp. $\mathcal{L}_{\square}$ 's) schemata $\left(S_{1}\right), \ldots,\left(S_{n}\right)$, if all $\mathcal{L}$ 's (resp. $\mathcal{L}_{\square}$ 's) formulas instantiating schemata $\left(S_{1}\right), \ldots,\left(S_{n}\right)$ are elements of the set $\Sigma$.
} 
$\left(\mathrm{K}^{\supset}\right) ; \Sigma$ is closed under the introduced modification of Disjunctive Syllogism and the Necessitation Rule, i.e.:

$$
\text { if } A \in \Sigma \text { then } \square A \in \Sigma \text {. }
$$

As in the case of axiomatic systems, any modal axiomatic system is closed under Modus Ponens.

The intended axiomatic system for $\mathrm{BC}_{\square}$ is the system $\mathrm{S}_{\square}$ that is the least modal axiomatic system. The intended axiomatic system for $\mathrm{BC}_{\square} \mathrm{X}_{1} \ldots \mathrm{X}_{n}$ is the system $\mathrm{S}_{\square} \mathrm{X}_{1} \ldots \mathrm{X}_{n}$ that is the least modal axiomatic system that contains $\mathcal{L}_{\square}$ 's schemata $\left(\mathrm{X}_{1}\right), \ldots,\left(\mathrm{X}_{n}\right)$.

Let $\Sigma$ be an axiomatic system and $\Gamma \cup\{A\} \subseteq$ For (resp. $\Gamma \cup\{A\} \subseteq$ For $\square$, for modal axiomatic systems). We have the following definition of the notion of a thesis and syntactic consequence:

- $A$ is a thesis (of $\Sigma$ ) iff $A \in \Sigma$

- $A$ is a syntactic consequence (in $\Sigma$ ) of $\Gamma$ (in symb.: $\Gamma \vdash_{\Sigma} A$ ) iff there is a set $\left\{B_{1}, \ldots, B_{n}\right\} \subseteq \Gamma(n \geq 0)$ such that $\left(B_{1} \wedge \cdots \wedge B_{n}\right) \supset A \in \Sigma .{ }^{10}$

By these definitions we get the following standard fact:

Proposition 4.1. Let $\Sigma$ be an axiomatic system and $\Gamma \cup\{A\} \subseteq$ For $(\Gamma \cup\{A\} \subseteq$ For $\square$, for modal axiomatic systems). Then, if $A \in \Sigma$ then $\Gamma \vdash_{\Sigma} A$.

\section{2. $\Sigma$-Consistent and Maximal $\Sigma$-Consistent Sets}

In this section we recall some well-known metalogical definitions and facts concerning consistent and maximal consistent sets defined with respect to an axiomatic system (cf. [1, pp. 47-49, 53-57]). Let $\Sigma$ be an axiomatic system (resp. a modal axiomatic system) and $\Gamma \subseteq$ For (resp. $\Gamma \subseteq$ For $_{\square}$ ). Then:

- $\Gamma$ is $\Sigma$-consistent iff $\Gamma \forall \Sigma p_{0} \wedge \neg p_{0}$

- $\Gamma$ is $\Sigma$-inconsistent iff $\Gamma$ is not $\Sigma$-consistent.

The following fact expresses some properties of $\Sigma$-consistent sets (cf. [1, pp. 47-48, theorem 2.16], [3, pp. 107-108, theorems 32.7-32.9]):

Proposition 4.2. Let $\Sigma$ be an axiomatic system (resp. a modal axiomatic system) and $\Gamma \cup\{A\} \subseteq$ For (resp. $\Gamma \cup\{A\} \subseteq$ For $\square$ ). Then:

1. $\Gamma \cup\{\neg A\}$ is $\Sigma$-consistent iff $\Gamma \forall \Sigma A$

2. if $A \notin \Sigma$ then $\{\neg A\} \forall \forall_{\Sigma} A$.

For modal axiomatic systems we also have the following proposition (cf. [2, p. 117, lemma 6.4]):

Proposition 4.3. Let $\Sigma$ be a modal axiomatic system and $\Gamma \cup\{A\} \subseteq$ For $\square$. Then, if $\Gamma$ is $\Sigma$-consistent and $\neg \square A \in \Gamma$, then $\left\{B \in\right.$ For $\left._{\square}: \square B \in \Gamma\right\} \cup\{\neg A\}$ is $\Sigma$-consistent.

\footnotetext{
${ }^{10}$ In the case of formulas built by an iteration of $\wedge$ we shall understand a left-most priority nesting of brackets, thus writing $A_{1} \wedge A_{2} \wedge A_{3} \wedge A_{4}$ instead of $\left(\left(A_{1} \wedge A_{2}\right) \wedge A_{3}\right) \wedge A_{4}$. In the case of an iteration $A_{1} \wedge \cdots \wedge A_{n}$ for $n=0$ we identify a sequence $\left(A_{1} \wedge \cdots \wedge A_{n}\right) \supset B$ with $B$.
} 
Proof. By $\left(\mathrm{K}^{\supset}\right)$ and $(\mathrm{NR})$, if $\left(A_{1} \wedge \cdots \wedge A_{n}\right) \supset B \in \Sigma$ then $\left(\square A_{1} \wedge \cdots \wedge \square A_{n}\right) \supset$ $\square B \in \Sigma$. Suppose $\{B \in$ For $\square: \square B \in \Gamma\} \cup\{\neg A\}$ is $\Sigma$-inconsistent. Hence, by fact 4.2 (1), $\{B \in$ For $\square: \square B \in \Gamma\} \vdash_{\Sigma} A$. By definition of syntactic consequence $\left(B_{1} \wedge \cdots \wedge B_{n}\right) \supset A \in \Sigma$, for some $n \in \mathbb{N}$ such that $\left\{B_{1}, \ldots, B_{n}\right\} \subseteq$ $\left\{B \in\right.$ For $\left._{\square}: \square B \in \Gamma\right\}$. Thus $\left(\square B_{1} \wedge \cdots \wedge \square B_{n}\right) \supset \square A \in \Sigma$. Since PL $\square \subseteq \Sigma$, by fact 4.1 , we get $\Gamma \vdash_{\Sigma} \square A \wedge \neg \square A$ and so $\Gamma \vdash_{\Sigma} p_{0} \wedge \neg p_{0}$. Hence, by the definition of a $\Sigma$-consistent set, $\Gamma$ is $\Sigma$-inconsistent.

The notion of maximal $\Sigma$-consistent set is defined in the standard way. Let $\Sigma$ be an axiomatic system (resp. a modal axiomatic system) and $\Gamma \subseteq$ For (resp. $\Gamma \subseteq$ For $_{\square}$ ). $\Gamma$ is maximal $\Sigma$-consistent iff the following conditions are satisfied:

- $\Gamma$ is $\Sigma$-consistent

- for every $\Delta \subseteq$ For ( $\Delta \subseteq$ For $_{\square}$, for modal axiomatic systems), if $\Gamma \subset \Delta$ then $\Delta$ is $\Sigma$-inconsistent.

The set of all maximal $\Sigma$-consistent sets is denoted by Max $x_{\Sigma}$. Elements of Max $x_{\Sigma}$ are theories in the following sense (cf. [1, p. 53, theorem 2.18 (1)], [3, p. 108, theorem 32.10]):

Proposition 4.4. Let $\Sigma$ be an axiomatic system, $\Gamma \in \operatorname{Max}_{\Sigma}$ and $A \in$ For $(A \in$ For $\square$, for modal axiomatic systems). Then, $A \in \Gamma$ iff $\Gamma \vdash_{\Sigma} A$.

Therefore, by means of facts 4.1, 4.4 we get the following corollary:

Corollary 4.5. Let $\Sigma$ be an axiomatic system (resp. a modal axiomatic system) and $\Gamma \in \operatorname{Max}_{\Sigma}$. Then $\Sigma \subseteq \Gamma$.

By means of fact 4.4 and the definitions of an axiomatic system and syntactic consequence we can prove the next standard fact that characterises connectives $\neg, \wedge, \vee$ on the basis of maximal $\Sigma$-consistent sets (cf. [1, p. 53, theorem $2.18(5)-(7)])$.

Proposition 4.6. Let $\Sigma$ be an axiomatic system (resp. a modal axiomatic system) and $\Gamma \in \operatorname{Max}_{\Sigma}$. Then, for every $A, B \in$ For (resp. $A, B \in$ For $_{\square}$ ):

1. $\neg A \in \Gamma$ iff $A \notin \Gamma$

2. $A \wedge B \in \Gamma$ iff $[A \in \Gamma$ and $B \in \Gamma]$

3. $A \vee B \in \Gamma$ iff $[A \in \Gamma$ or $B \in \Gamma]$.

The next fact is widely known as Lindenbaum's lemma (cf. [1, p. 55, theorem 2.19], [3, p. 110, theorem 3.12]), we use it in our proofs of completeness:

Proposition 4.7. Let $\Sigma$ be an axiomatic system (resp. a modal axiomatic system) and $\Gamma \subseteq$ For (resp. $\Gamma \subseteq$ For $_{\square}$ ). Then, if $\Gamma$ is $\Sigma$-consistent, then there is $\Delta \subseteq$ For (resp. $\Delta \subseteq$ For $_{\square}$ ) such that $\Gamma \subseteq \Delta$ and $\Delta \in \operatorname{Max}_{\Sigma}$. 


\subsection{Canonical Models}

Let us now present the notion of the canonical model defined by means of maximal $\Sigma$-consistent set (cf. [1, pp. 59-62], [3, pp. 111-112], [2, pp. 101-104]). We first deal with canonical models for $\mathcal{L}$.

Let $\Sigma$ be an axiomatic system and $\Gamma \in \operatorname{Max}_{\Sigma}$. The canonical model for $\mathcal{L}$ generated by $\Gamma$ (for short: $\Gamma$-model for $\mathcal{L}$ ) is a pair $\langle v, R\rangle$ such that:

- for every $A \in \operatorname{Var}$ :

$$
v(A)= \begin{cases}1, & \text { if } A \in \Gamma \\ 0, & \text { if } A \notin \Gamma\end{cases}
$$

- for every $A, B \in$ For:

$$
R(A, B) \text { iff } A \rightarrow B \in \Gamma .
$$

We have the following lemma:

Lemma 4.8. Let $\Sigma$ be an axiomatic system, $\Gamma \in \operatorname{Max}_{\Sigma}$ and $\mathfrak{M}$ be the $\Gamma$-model for $\mathcal{L}$. Then, for every $A \in$ For, $\mathfrak{M}=A$ iff $A \in \Gamma$.

Proof. Assume all the hypotheses. We use induction on the complexity of formulas from For.

Base case. Let $A \in$ For and $\mathrm{c}(A)=0$. Then $\mathfrak{M} \models A$ iff $v_{\mathfrak{M}}(A)=1$, by definition of $v_{\mathfrak{M}}$, iff $A \in \Gamma$.

Inductive hypothesis. Let $n \in \mathbb{N}$. Suppose for every $A \in$ For, if $\mathrm{c}(A) \leq n$, then $\mathfrak{M} \models A$ iff $A \in \Gamma$.

Inductive step. Let $A \in$ For and $\mathrm{c}(A)=n+1$. In the following cases $A=\neg B, A=B \wedge C, A=B \vee C$ we reason in the standard way using the inductive hypothesis and fact 4.6. Let $A=B \rightarrow C$.

" $\Rightarrow$ " Suppose $\mathfrak{M}=A$. Thus $\mathfrak{M}=B \rightarrow C$ and by the definition of truth in a model $R_{\mathfrak{M}}(B, C)$. Hence, by the definition of $\Gamma$-model for $\mathcal{L}, B \rightarrow C \in \Gamma$. Therefore $A \in \Gamma$.

" $\Leftarrow$ " Suppose $A \in \Gamma$. Thus $B \rightarrow C \in \Gamma$. Hence, by the definition of canonical model for $\mathrm{BC}, R_{\mathfrak{M}}(B, C)$. By corollary $4.5 \Gamma$ contains $\mathcal{L}$ 's schema (Imp). Hence, by fact 4.61 and $3, B \supset C \in \Gamma$. By fact 4.61 and 3 , either $B \notin \Sigma$ or $C \in \Gamma$. By the inductive hypothesis either $\mathfrak{M} \forall B$ or $\mathfrak{M} \models C$. Therefore, since $R_{\mathfrak{M}}(B, C), \mathfrak{M} \models B \rightarrow C$, and so $\mathfrak{M} \models A$.

Notice that the canonical model for $\mathcal{L}$ generated by $\Gamma$ is a model from the class MC, i.e. the class of all models over connexive relations between formulas.

Proposition 4.9. Let $\Sigma$ be an axiomatic system and $\Gamma \in \operatorname{Max}_{\Sigma}$. Then, if $\mathfrak{M}$ is the $\Gamma$-model for $\mathcal{L}$, then $\mathfrak{M} \in M C$.

Proof. Assume all the hypotheses. Let $\mathfrak{M}$ be the $\Gamma$-model for $\mathcal{L}$.

For (a1), (a2). By corollary $4.5 \Gamma$ contains $\mathcal{L}$ 's schemata (A1), (A2). By the definition of $\Gamma$-model for $\mathcal{L} \sim R_{\mathfrak{M}}(A, \neg A)$ and $\sim R_{\mathfrak{M}}(\neg A, A)$, for every $A \in$ For. 
For (b0). By corollary $4.5 \Gamma$ contains $\mathcal{L}$ 's schema (B1). Let $A, B \in$ For and suppose $R_{\mathfrak{M}}(A, B)$. Thus, by the definition of the $\Gamma$-model for $\mathcal{L}, A \rightarrow B \in \Gamma$. Since $\Gamma$ also contains $\mathcal{L}$ 's schema $(\operatorname{Imp}),(A \rightarrow B) \supset \neg(A \rightarrow \neg B) \in \Gamma$. Hence, by fact 4.61 and $3, A \rightarrow \neg B \notin \Gamma$. Thus, by the definition of the $\Gamma$-model for $\mathcal{L}, \sim R_{\mathfrak{M}}(A, \neg B) \in \Gamma$.

For (b1), (b2). By corollary $4.5 \Gamma$ contains $\mathcal{L}$ 's schemata (B1), (B2). By the definition of $\Gamma$-model for $\mathcal{L} R_{\mathfrak{M}}(A \rightarrow B, \neg(A \rightarrow \neg B))$ and $R_{\mathfrak{M}}(A \rightarrow \neg B$, $\neg(A \rightarrow B))$, for every $A, B \in$ For.

In the case of the axiomatic system $\mathrm{S}\urcorner$, we produce a new relation between formulas based on a given canonical model. For schemata $\left(\mathrm{Cun}_{1}\right)$ and $\left(\mathrm{Cun}_{2}\right)$ are not sufficient to prove that relations of canonical models of the kind defined above are closed under negation.

Let $\Sigma \in \operatorname{Max} \neg$ and $\mathfrak{M}$ be a $\Sigma$-model. Relation $R_{\mathfrak{M}}^{\neg}$ is the least relation among those relations $Q \subseteq$ For $\times$ For that satisfy the conditions:

$$
\begin{gathered}
\text { if } \mathfrak{M} \models A \rightarrow B \text {, then } Q(A, B) \\
\text { if } \mathfrak{M} \models A \rightarrow B \text { and } \mathfrak{M} \models \neg A \wedge B \text {, then } Q(\neg A, \neg B) .
\end{gathered}
$$

Let us observe that if $R_{\mathfrak{M}}^{\neg}(A, B)$, then either $\mathfrak{M} \models A \rightarrow B$ or $A=\neg A^{\prime}$, $B=\neg B^{\prime}, \mathfrak{M} \models A^{\prime} \rightarrow B^{\prime}$ and $\mathfrak{M}=\neg A^{\prime} \wedge B^{\prime}$. Otherwise $R_{\mathfrak{M}}^{\neg}$ would not be the least relation that satisfies $\left(\mathrm{rel}_{1}\right)$ and $\left(\mathrm{rel}_{2}\right)$. What is important is that the change of the relation of the canonical model on the new one received by the canonical model and conditions $\left(\mathrm{rel}_{1}\right),\left(\mathrm{rel}_{2}\right)$ does not change the theory of the model.

Lemma 4.10. Let $\Sigma \in \mathrm{Maxs}\urcorner$ and $\mathfrak{M}$ be the $\Sigma$-model for $\mathcal{L}$. Then, for every $A \in$ For, $\mathfrak{M} \models A$ iff $\left\langle v_{\mathfrak{M}}, R_{\mathfrak{M}}^{\neg}\right\rangle \models A$.

Proof. Assume all the hypotheses. We use induction on the complexity of formulas from For.

Base case. Let $A \in$ For and $\mathrm{c}(A)=0$. Then $\mathfrak{M} \models A$ iff $v_{\mathfrak{M}}(A)=1$ iff $\left\langle v_{\mathfrak{M}}, R_{\mathfrak{M}}^{\neg}\right\rangle \models A$.

Inductive hypothesis. Let $n \in \mathbb{N}$. Suppose for every $A \in$ For, if $\mathrm{c}(A) \leq n$, then $\mathfrak{M}=A$ iff $A \in \Gamma$.

Inductive step. Let $A \in$ For and $\mathrm{c}(A)=n+1$. In the following cases $A=\neg B, A=B \wedge C, A=B \vee C$ we reason in the standard way using the inductive hypothesis. Let $A=B \rightarrow C$.

" $\Rightarrow$ " Suppose $\mathfrak{M}=B \rightarrow C$. Thus, by the definition of truth in a model, $\mathfrak{M} \not \models B$ or $\mathfrak{M} \models C$ and $R_{\mathfrak{M}}(B, C)$. Hence, by the inductive hypothesis, $\left\langle v_{\mathfrak{M}}, R_{\mathfrak{M}}^{\neg}\right\rangle \not \models B$ or $\left\langle v_{\mathfrak{M}}, R_{\mathfrak{M}}^{\neg}\right\rangle \models C$. And since $\mathfrak{M} \models B \rightarrow C$, by the definition of $R_{\mathfrak{M}}^{\neg}, R_{\mathfrak{M}}^{\neg}(B, C)$. Thus, by the definition of truth in a model, $\left\langle v_{\mathfrak{M}}, R_{\mathfrak{M}}^{\neg}\right\rangle=B \rightarrow C$.

"६" Suppose $\left\langle v_{\mathfrak{M}}, R_{\mathfrak{M}}^{\neg}\right\rangle=B \rightarrow C$. Thus, by the definition of truth in a model, $\left\langle v_{\mathfrak{M}}, R_{\mathfrak{M}}^{\neg}\right\rangle \not \models B$ or $\left\langle v_{\mathfrak{M}}, R_{\mathfrak{M}}^{\neg}\right\rangle \models C$ and $R_{\mathfrak{M}}^{\neg}(B, C)$. By the definition of $R_{\mathfrak{M}}^{\neg}$ either (1) $\mathfrak{M} \models B \rightarrow C$ or $(2) B=\neg B^{\prime}, C=\neg C^{\prime}, \mathfrak{M} \models B^{\prime} \rightarrow C^{\prime}$ and $\mathfrak{M}=\neg B^{\prime} \wedge C^{\prime}$. By the inductive hypothesis either $\mathfrak{M} \not \models B$ or $\mathfrak{M} \mid=C$. Thus either $\mathfrak{M} \not \neq \neg B^{\prime}$ or $\mathfrak{M} \not \models C^{\prime}$. Hence (2) cannot hold. By (1) $\mathfrak{M} \models B \rightarrow C$. 
We can show that the model received from the canonical model for $\mathcal{L}$ generated by $\Sigma \in$ Maxs $\neg$ by means of $\left(\operatorname{rel}_{1}\right),\left(\mathrm{rel}_{2}\right)$ is a model from the class $\mathbf{M C}^{\urcorner}$:

Proposition 4.11. Let $\Sigma \in \mathrm{Max}_{\mathrm{S}}$. Then, if $\mathfrak{M}$ is the $\Sigma$-model for $\mathcal{L}$ then $\left.\left\langle v_{\mathfrak{M}}, R_{\mathfrak{M}}^{\neg}\right\rangle \in M C\right\urcorner$.

Proof. Assume the hypothesis. Let $\mathfrak{M}$ be the $\Sigma$-model for $\mathcal{L}$.

For (cun). Let $A, B \in$ For and suppose $R_{\mathfrak{M}}^{\neg}(A, B)$. Thus, either (1) $\mathfrak{M} \models$ $A \rightarrow B$ or $(2) A=\neg A^{\prime}, B=\neg B^{\prime}, \mathfrak{M} \models A^{\prime} \rightarrow B^{\prime}$ and $\mathfrak{M} \models \neg A^{\prime} \wedge B^{\prime}$.

Suppose (1) holds. By corollary $4.5 \Sigma$ contains $\mathcal{L}^{\prime}$ 's schema $\left(\mathrm{Cun}_{1}\right)$. Hence, by lemma 4.8, $\mathfrak{M} \models(A \rightarrow B) \supset((\neg A \rightarrow \neg B) \vee(\neg A \wedge B))$. Then, by the definition of truth in a model, either $\mathfrak{M} \models \neg A \rightarrow \neg B$ or $\mathfrak{M} \models \neg A \wedge B$. Thus, by definition of $R_{\mathfrak{M}}^{\neg}, R_{\mathfrak{M}}^{\neg}(\neg A, \neg B)$.

Suppose (2) holds. By corollary $4.5 \Sigma$ contains $\mathcal{L}^{\prime}$ 's schema $\left(\mathrm{Cun}_{2}\right)$. Hence, by lemmas $4.8, \mathfrak{M} \models\left(A^{\prime} \rightarrow B^{\prime}\right) \supset\left(\neg \neg A^{\prime} \rightarrow \neg \neg B^{\prime}\right)$. Then, by the definition of truth in a model, $\mathfrak{M} \models \neg \neg A^{\prime} \rightarrow \neg \neg B^{\prime}$ and $\mathfrak{M} \models \neg \neg \neg A^{\prime} \wedge \neg \neg B^{\prime}$. Thus, by definition of $R_{\mathfrak{M}}^{\neg}, R_{\mathfrak{M}}^{\neg}(\neg A, \neg B)$.

For (a1). By corollary $4.5 \Sigma$ contains $\mathcal{L}$ 's schema (A1). Let $A \in$ For. Thus, by lemma 4.8, $\mathfrak{M} \forall A \rightarrow \neg A$ and $\mathfrak{M} \not \models \neg A \rightarrow \neg \neg A$. By lemma 4.10 $\left\langle v_{\mathfrak{M}}, R_{\mathfrak{M}}^{\neg}\right\rangle \not \forall A \rightarrow \neg A$ and $\left\langle v_{\mathfrak{M}}, R_{\mathfrak{M}}^{\neg}\right\rangle \not \forall \neg A \rightarrow \neg \neg A$. Thus, by the definition of truth in a model, either $(1)\left\langle v_{\mathfrak{M}}, R_{\mathfrak{M}}^{\neg}\right\rangle \models A$ or $(2) \sim R_{\mathfrak{M}}^{\neg}(A, \neg A)$.

Suppose (1) holds and $R_{\mathfrak{M}}^{\neg}(A, \neg A)$. Since $R_{\mathfrak{M}}^{\neg}$ is closed under negation, so $R_{\mathfrak{M}}^{\neg}(\neg A, \neg \neg A)$. Thus, by the definition of truth in a model, $\left\langle v_{\mathfrak{M}}, R_{\mathfrak{M}}^{\neg}\right\rangle \models \neg \neg A$. Thus $\left\langle v_{\mathfrak{M}}, R_{\mathfrak{M}}^{\neg}\right\rangle \models \neg A \rightarrow \neg \neg A$. We get a contradiction.

For (a2). Similarly as for (a1).

For (b0). Suppose that $R_{\mathfrak{M}}^{\neg}$ does not satisfy (b0). Thus $R_{\mathfrak{M}}^{\neg}(A, B)$ and $R_{\mathfrak{M}}^{\neg}(A, \neg B)$, for some $A, B \in$ For.

Suppose $\left\langle v_{\mathfrak{M}}, R_{\mathfrak{M}}^{\neg}\right\rangle \models A$. Since $R_{\mathfrak{M}}^{\neg}$ is closed under negation, so $R_{\mathfrak{M}}^{\neg}(\neg A$, $\neg B)$ and $R_{\mathfrak{M}}(\neg A, \neg \neg B)$. Then, by the definition of truth in a model, $\left\langle v_{\mathfrak{M}}\right.$, $\left.R_{\mathfrak{M}}^{\neg}\right\rangle \models \neg A \rightarrow \neg B$ and $\left\langle v_{\mathfrak{M}}, R_{\mathfrak{M}}^{\neg}\right\rangle \models \neg A \rightarrow \neg \neg B$. By lemma $4.10 \mathfrak{M} \models \neg A \rightarrow$ $\neg B$ and $\mathfrak{M} \models \neg A \rightarrow \neg \neg B$. By corollary $4.5 \Sigma$ contains $\mathcal{L}$ 's schema (B1). Hence, by lemma 4.8, $\mathfrak{M} \models(\neg A \rightarrow \neg B) \rightarrow \neg(\neg A \rightarrow \neg \neg B)$. Thus, by the definition of truth in a model, either $\mathfrak{M} \not \models \neg A \rightarrow \neg B$ or $\mathfrak{M} \forall \neg A \rightarrow \neg \neg B$. We get a contradiction.

Suppose $\left\langle v_{\mathfrak{M}}, R_{\mathfrak{M}}^{\neg}\right\rangle \not \models A$. Then, by the definition of truth in a model, $\left\langle v_{\mathfrak{M}}, R_{\mathfrak{M}}^{\neg}\right\rangle \models A \rightarrow B$ and $\left\langle v_{\mathfrak{M}}, R_{\mathfrak{M}}^{\neg}\right\rangle=A \rightarrow \neg B$. By lemma $4.10 \mathfrak{M} \models A \rightarrow B$ and $\mathfrak{M} \models A \rightarrow \neg B$. By corollary $4.5 \Sigma$ contains $\mathcal{L}$ 's schema (B1). Hence, by lemma 4.8, $\mathfrak{M} \models(A \rightarrow B) \rightarrow \neg(A \rightarrow \neg B)$. Thus, by the definition of truth in a model, either $\mathfrak{M} \forall A \rightarrow B$ or $\mathfrak{M} \forall A \rightarrow \neg B$. We get a contradiction.

For (b1). By corollary $4.5 \Sigma$ contains $\mathcal{L}$ 's schema (B1). Let $A, B \in$ For. Hence, by lemmas $4.8, \mathfrak{M} \models(A \rightarrow B) \rightarrow \neg(A \rightarrow \neg B)$. Thus, by lemma 4.10, $\left\langle v_{\mathfrak{M}}, R_{\mathfrak{M}}^{\neg}\right\rangle=(A \rightarrow B) \rightarrow \neg(A \rightarrow \neg B)$. Thus, by the definition of truth in a model, $R_{\mathfrak{M}}^{\neg}(A \rightarrow B, \neg(A \rightarrow \neg A))$.

For (b2). Similarly as for (b1).

By fact 4.7 and lemma 4.8 we get the following corollary: 
Corollary 4.12. Let $\Sigma$ be an axiomatic system and $\Gamma \subseteq$ For. Then, if $\Gamma$ is a $\Sigma$-consistent set, then there is $\Delta \in \operatorname{Max}_{\Sigma}$ such that $\mathfrak{M}$ is the $\Delta$-model for $\mathcal{L}$ and $\mathfrak{M}=\Gamma$.

Proof. Assume all the hypotheses. Suppose $\Gamma$ is $\Sigma$-consistent. By fact 4.7 there is $\Delta \in \operatorname{Max}_{\Sigma}$, such that $\Gamma \subseteq \Delta$. By lemma 4.8 , for every $A \in \Delta, \mathfrak{M} \models A$, where $\mathfrak{M}$ is the $\Delta$-model for $\mathcal{L}$. Hence $\mathfrak{M}=\Gamma$.

Thus we get the following completeness lemma:

Lemma 4.13. Let $\Sigma$ be an axiomatic system and $A \in$ For. Then, $A \in \Sigma$ iff for every $\Gamma \in \operatorname{Max}_{\Sigma}$, if $\mathfrak{M}$ is the $\Gamma$-model for $\mathcal{L}$, then $\mathfrak{M} \models A$.

Proof. Assume all the hypotheses.

" $\Rightarrow$ " Suppose $A \in \Sigma, \Gamma \in \operatorname{Max}_{\Sigma}$ and let $\mathfrak{M}$ be the $\Gamma$-model for $\mathcal{L}$. By corollary $4.5 A \in \Gamma$. Hence, by lemma $4.8, \mathfrak{M}=A$.

" $\Leftarrow$ " Suppose $A \notin \Sigma$. By fact $4.2\{\neg A\}$ is $\Sigma$-consistent. By fact 4.7 there is $\Gamma \in \operatorname{Max}_{\Sigma}$ such that $\{\neg A\} \subseteq \Gamma$, so $\neg A \in \Gamma$. By lemma $4.8 \mathfrak{M}=\neg A$, where $\mathfrak{M}$ is the $\Gamma$-model for $\mathcal{L}$. Therefore $\mathfrak{M}_{\Gamma} \not \models A$.

A canonical model for $\mathcal{L}_{\square}$ is the standard canonical model for modal language with a family of relations between formulas indexed by maximal consistent sets. Let $\Sigma$ be a modal axiomatic system. The canonical model for $\mathcal{L}_{\square}$ generated by $\operatorname{Max}_{\Sigma}$ (for short: $\operatorname{Max}_{\Sigma}$-model for $\mathcal{L}$ ) is a quadruple $\left\langle W, Q,\left\{R_{w}\right\}_{w \in W}, v\right\rangle$ such that:

- $W=\operatorname{Max}_{\Sigma}$

- for every $w, u \in W$ :

$Q(w, u)$ iff for every $A \in$ For $_{\square}$, if $\square A \in w$ then $A \in u$

- for every $w \in W$, for every $A, B \in$ For $_{\square}$ :

$$
R_{w}(A, B) \text { iff } A \rightarrow B \in w
$$

- for every $w \in W$, for every $A \in$ Var:

$$
v(w, A)= \begin{cases}1, & \text { if } A \in w \\ 0, & \text { if } A \notin w .\end{cases}
$$

Here we have the following standard lemma about canonical models for modal axiomatic systems:

Lemma 4.14. Let $\Sigma$ be a modal axiomatic system, $\mathfrak{M}$ be the Max $x_{\Sigma}$-model for $\mathcal{L}_{\square}$ and $w \in W_{\mathfrak{M}}$. Then, for every $A \in$ For, $\mathfrak{M}, w=A$ iff $A \in w$.

Proof. Assume all the hypotheses. We use induction on the complexity of formulas from For $\square$.

Base case. Let $A \in$ For $_{\square}$ and $\mathrm{c}(A)=0$. Then: $\mathfrak{M}, w=A$ iff $v_{\mathfrak{M}}(w, A)=1$, by the definition of $\operatorname{Max}_{\Sigma}$-model for $\mathcal{L}_{\square}$, iff $A \in w$.

Inductive hypothesis. Let $n \in \mathbb{N}$. Suppose for every $A \in$ For, if $\mathrm{c}(A) \leq n$, then $\mathfrak{M}, w \models A$ iff $A \in w$.

Inductive step. Let $A \in$ For and $\mathrm{c}(A)=n+1$. In the following cases: $A=\neg B, A=B \wedge C, A=B \vee C, A=\square B, A=\diamond B$ we reason in the standard 
way. In the case $A=\square B$, we use fact 4.3 , cf. [2, p. 118 , theorem 6.5$]$. In the case $A=\diamond B$ we refer to the case $A=\square B$, the schema (Dual $\equiv$ ) and corollary 4.5. Let $A=B \rightarrow C$.

" $\Rightarrow$ " Suppose $\mathfrak{M}, w \models A$. Thus $\mathfrak{M}, w \models B \rightarrow C$ and by the definition of truth in a model, in a world $R_{w}(B, C)$. Hence, by the definition of Max ${ }_{\Sigma}$-model for $\mathcal{L}_{\square}, B \rightarrow C \in w$. Therefore $A \in w$.

" $\Leftarrow$ " Suppose $A \in w$. Thus $B \rightarrow C \in w$. Hence, by the definition of $\operatorname{Max}_{\Sigma}$-model for $\mathcal{L}_{\square}, R_{w}(B, C)$. Since $\Sigma$ contains $\mathcal{L}_{\square}$ 's schema (Imp) and by fact 4.61 and 3,B $\supset C \in w$. Thus, again by the fact 4.61 and 3, either $B \notin w$ or $C \in w$. By the inductive hypothesis either $\mathfrak{M}, w \not \neq B$ or $\mathfrak{M}, w \models C$. Therefore, since $R_{w}(B, C), \mathfrak{M}, w \models B \rightarrow C$, and so $\mathfrak{M}, w \models A$.

Notice that any canonical model for $\mathcal{L}_{\square}$ is a model from the class $\mathbf{M C}_{\square}$.

Proposition 4.15. Let $\Sigma$ be a modal axiomatic system. Then, if $\mathfrak{M}$ is the $\mathrm{Max}_{\Sigma^{-}}$ model for $\mathcal{L}_{\square}$, then $\mathfrak{M} \in M C_{\square}$.

Proof. We reason similarly as in the proof of fact 4.9 .

By fact 4.15 we know that combined frames of canonical models for $\mathcal{L}_{\square}$ are elements of the class $\mathbf{C}_{\square}$. Now we prove that they are also elements of some proper subclasses of $\mathbf{C}_{\square}$, if the considered axiomatic systems contain some axiom schemata.

Lemma 4.16. Let $\Sigma$ be a modal axiomatic system and $\mathfrak{M}$ be the Max $x_{-}$-model for $\mathcal{L}_{\square}$ and $\mathrm{X} \in\left\{\mathrm{D}_{1}, \mathrm{D}_{2}, \mathrm{~K}, \mathrm{~T}, \mathrm{D}, \mathrm{B}, 4,5\right\}$. Then, if $\Sigma$ contains $\mathcal{L}_{\square}$ 's schema (X), then $\mathfrak{M} \in M C_{\square} \mathbf{X}$.

Proof. Assume all the hypotheses. Let us consider only cases for $\mathrm{X} \in\left\{\mathrm{D}_{1}, \mathrm{~K}\right.$, $\mathrm{T}\}$, as the rest of the cases we can reason in a similar way.

For $\mathrm{X}=\mathrm{D}_{1}$. By corollary $4.5 w \in W_{\mathfrak{M}}$ contains $\mathcal{L}_{\square}$ 's schema $\left(\mathrm{D}_{1}\right)$. Thus, by the definition of Max ${ }_{\Sigma}$-model for $\mathcal{L}_{\square}, R_{w}(\diamond A, \neg \square \neg A)$, for every $A \in$ For $_{\square}$. Therefore $\left\langle W_{\mathfrak{M}}, Q_{\mathfrak{M}},\left\{R_{w}\right\}_{w \in W_{\mathfrak{M}}}\right\rangle \in \mathbf{C}_{\square} \mathbf{D}_{1}$.

For $\mathrm{X}=\mathrm{K}$. By corollary $4.5 w \in W_{\mathfrak{M}}$ contains $\mathcal{L}_{\square}$ 's schema $(\mathrm{K})$. Thus, by the definition of Max ${ }_{\Sigma}$-model for $\mathcal{L}_{\square}, R_{w}(\square(A \rightarrow B), \square A \rightarrow \square B)$, for every $A, B \in$ For $_{\square}$. Let $A, B \in$ For $\square$ and suppose, for every $u \in W_{\mathfrak{M}}$, if $Q_{\mathfrak{M}}(w, u)$

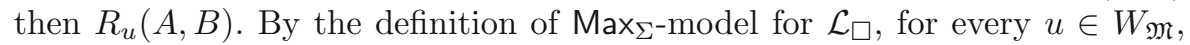
if $Q_{\mathfrak{M}}(w, u)$ then $A \rightarrow B \in u$. Thus, by lemma 4.13, for every $u \in W_{\mathfrak{M}}$, if $Q_{\mathfrak{M}}(w, u)$ then $\mathfrak{M}, u \models A \rightarrow B$. Hence, by the definition of truth in a model, in a world, $\mathfrak{M}, w \models \square(A \rightarrow B)$. By lemma $4.13 \square(A \rightarrow B) \in w$. By corollary $4.5 w$ contains $\mathcal{L}_{\square}$ 's schema $\left(\mathrm{K}^{\supset}\right)$. Hence, by fact 4.61 and 3 , $\square A \rightarrow \square B \in w$. Thus, by the definition of Max $\Sigma_{\Sigma}$-model for $\mathcal{L}_{\square}, R_{w}(\square A, \square B)$. Therefore $\left\langle W_{\mathfrak{M}}, Q_{\mathfrak{M}},\left\{R_{w}\right\}_{w \in W_{\mathfrak{M}}}\right\rangle \in \mathbf{C}_{\square} \mathbf{K}$.

For $\mathrm{X}=\mathrm{T}$. By corollary $4.5 w \in W_{\mathfrak{M}}$ contains $\mathcal{L}_{\square}$ 's schema $(\mathrm{T})$. Thus, by the definition of Max ${ }_{\Sigma}$-model for $\mathcal{L}_{\square}, R_{w}(\square A, A)$, for every $A \in$ For $\square$. Since $w$ contains $\mathcal{L}_{\square}$ 's schemata $(\mathrm{T}),(\operatorname{Imp})$, then by fact 4.61 and 3 , if $\square A \in w$ then $A \in w$, for every $A \in$ For $_{\square}$. Thus, by the definition of Max Thodel for $^{-m}$ $\mathcal{L}_{\square}, Q_{\mathfrak{M}}(w, w)$. Therefore $\left\langle W_{\mathfrak{M}}, Q_{\mathfrak{M}},\left\{R_{w}\right\}_{w \in W_{\mathfrak{M}}}\right\rangle \in \mathbf{C}_{\square} \mathbf{T}$.

By lemma 4.16 we receive the following corollary: 
Corollary 4.17. Let $\Sigma$ be a modal axiomatic system and $\mathfrak{M}$ be the Max $\mathrm{x}_{\Sigma}$-model for $\mathcal{L}_{\square}, n>0$ and for every $i \leq n, \mathrm{X}_{i} \in\left\{\mathrm{D}_{1}, \mathrm{D}_{2}, \mathrm{~K}, \mathrm{~T}, \mathrm{D}, \mathrm{B}, 4,5\right\}$. Then, if $\Sigma$ contains $\mathcal{L}_{\square}$ 's schemata $\left(\mathrm{X}_{1}\right), \ldots,\left(\mathrm{X}_{n}\right)$ then $\mathfrak{M} \in M C_{\square} \mathbf{X}_{i} \ldots \mathbf{X}_{n}$.

By fact 4.7 and lemma 4.14 we get the following corollary:

Corollary 4.18. Let $\Sigma$ be an axiomatic system and $\Gamma \subseteq$ For. Then, if $\Gamma$ is a $\Sigma$-consistent set, then there is a $w \in \operatorname{Max}_{\Sigma}$ such that $\mathfrak{M}$ is the $\operatorname{Max}_{\Sigma}$-model for $\mathcal{L}_{\square}$ and $\mathfrak{M}, w \models \Gamma$.

Proof. Assume all the hypotheses. Suppose $\Gamma$ is $\Sigma$-consistent. By fact 4.7 there is a $w \in \operatorname{Max}_{\Sigma}$, such that $\Gamma \subseteq w$. By lemma 4.14, for every $A \in w, \mathfrak{M}, w=A$. Hence $\mathfrak{M}, w \models \Gamma$.

By lemma 4.14 and corollary 4.18 we get the following completeness lemma (cf. [2, p. 119, corollary 6.6]):

Lemma 4.19. Let $\Sigma$ is a modal axiomatic system, $\mathfrak{M}$ be the Max ${ }_{\Sigma}$-model for $\mathcal{L}_{\square}$ and $A \in$ For $_{\square}$. Then, $A \in \Sigma$ iff for every $w \in W_{\mathfrak{M}}, \mathfrak{M}, w \models A$.

Proof. Assume all the hypotheses.

" $\Rightarrow$ " Suppose $A \in \Sigma$. Let $w \in W_{\mathfrak{M}}=\operatorname{Max}_{\Sigma}$. By corollary $4.5 A \in w$. By lemma $4.14 \mathfrak{M}, w \models A$.

"£" Suppose $A \notin \Sigma$. Hence, by fact $4.2,\{\neg A\}$ is $\Sigma$-consistent. By corollary 4.18 there is $w \in \operatorname{Max}_{\Sigma}$ such that $\mathfrak{M}$ is the Max ${ }_{\Sigma}$-model for $\mathcal{L}_{\square}$ and $\mathfrak{M}, w \models \neg A$. Therefore $\mathfrak{M}, w \not \models A$.

\section{Soundness and Completeness}

We first deal with the soundness and completeness theorem for systems $\mathrm{S}$ and $\mathrm{S}\urcorner$.

Theorem 5.1. Let $\Sigma \cup\{A\} \subseteq$ For. Then:

1. $A \in \mathrm{S}$ iff $A \in \mathrm{BC}$

2. $A \in \mathrm{S}\urcorner$ iff $A \in \mathrm{BC}\urcorner$.

Proof. Assume all the hypotheses.

Ad 1. " $\Rightarrow "$ Obviously PL $\subseteq$ BC, BC is closed under the introduced modification of Disjunctive Syllogism and contains $\mathcal{L}$ 's schemata (Imp), (A1), (A2), (B1), (B2), since all of them are valid in the class $\mathbf{C}$ (see $[8$, p. 435, theorem $5.1])$.

"ఓ" Suppose $A \in \mathrm{BC}$. Hence, by fact $3.1, \mathfrak{M} \models A$, for every $\mathfrak{M} \in \mathbf{M C}$. Thus, by fact 4.9 , for every $\Sigma \in$ Maxs, if $\mathfrak{M}$ is the $\Sigma$-model for $\mathcal{L}$, then $\mathfrak{M} \models A$. Therefore, by lemma $4.13, A \in \mathrm{S}$.

Ad 2. Similarly as ad 1 . For the proof of " $\Rightarrow$ ", obviously BC $\urcorner$ contains $\mathcal{L}$ 's schemata $\left(\mathrm{Cun}_{1}\right),\left(\mathrm{Cun}_{2}\right)$, since they are valid in the class $\left.\mathbf{C}\right\urcorner$ of the relations closed under negation. In the proof of " $\Leftarrow$ " we use fact 4.11 instead of 4.9 and lemma 4.10. 
Similarly, we can prove soundness and completeness for modal axiomatic systems:

Theorem 5.2. Let $\Sigma \cup\{A\} \subseteq$ For $\square$. Then:

1. $A \in \mathrm{S}_{\square}$ iff $A \in \mathrm{BC}_{\square}$

2. $A \in \mathrm{S}_{\square} \mathrm{X}_{1} \ldots \mathrm{X}_{n}$ iff $A \in \mathrm{BC}_{\square} \mathrm{X}_{1} \ldots \mathrm{X}_{n}$, where for every $0<i \leq n$, $\mathrm{X}_{i} \in\left\{\mathrm{D}_{1}, \mathrm{D}_{2}, \mathrm{~K}, \mathrm{~T}, \mathrm{D}, \mathrm{B}, 4,5\right\}$.

Proof. Assume all the hypotheses.

Ad 1. " $\Rightarrow$ " Obviously PL $\square \subseteq \mathrm{BC}_{\square}$ and $\mathrm{BC}_{\square}$ is closed under the introduced modification of Disjunctive Syllogism. As we noticed above $\mathrm{BC}_{\square}$ is closed under Necessitation Rule, and it contains $\mathcal{L}_{\square}$ 's schemata (Imp), (Dual $\left.{ }^{\equiv}\right),\left(\mathrm{K}^{\supset}\right),(\mathrm{A} 1)$, (A2), (B1), (B2), since of all of them are valid in the class $\mathbf{C}_{\square}$.

" $\Leftarrow$ " Suppose $A \in \mathrm{BC}_{\square}$. Hence, by fact $3.2, \mathfrak{M}, w=A$, for every $\mathfrak{M} \in \mathbf{M C}$ and any $w \in W_{\mathfrak{M}}$. Thus, by fact 4.15 , if $\mathfrak{M}$ is the Max $\mathrm{s}_{\square}$-model for $\mathcal{L}_{\square}$, then for every $w \in W_{\mathfrak{M}}, \mathfrak{M}, w \models A$. Therefore, by lemma $4.19, A \in \mathrm{S}_{\square}$.

Ad 2. Similarly as ad 1 . In the proof of " $\Rightarrow$ " we refer to $[9$, p. 222 , theorem 4.2 ; pp. $224-225$, claim 5.2 ; pp. $225-226$, claim 5.3$]$. In the proof of " $\Leftarrow$ " we use corollary 4.17 .

\section{Acknowledgements}

This work was supported by the National Science Centre, Poland, under Grants UMO-2015/19/N/HS1/02401. I also would like to thank Tomasz Jarmużek for all his advice and remarks on this article.

Open Access. This article is licensed under a Creative Commons Attribution 4.0 International License, which permits use, sharing, adaptation, distribution and reproduction in any medium or format, as long as you give appropriate credit to the original author(s) and the source, provide a link to the Creative Commons licence, and indicate if changes were made. The images or other third party material in this article are included in the article's Creative Commons licence, unless indicated otherwise in a credit line to the material. If material is not included in the article's Creative Commons licence and your intended use is not permitted by statutory regulation or exceeds the permitted use, you will need to obtain permission directly from the copyright holder. To view a copy of this licence, visit http://creativecommons. org/licenses/by/4.0/.

Publisher's Note Springer Nature remains neutral with regard to jurisdictional claims in published maps and institutional affiliations.

\section{References}

[1] Chellas, B.F.: Modal Logic. An Introduction. Cambridge University Press (1980)

[2] Hughes, G.E., Cresswell, M.J.: A New Introduction to Modal Logic. Routledge (1996)

[3] Hunter, G.: Metalogic. An Introduction to the Metatheory of Standard First Order Logic. University of California Press (1973) 
[4] Jarmużek, T.: Relating semantics as fine-grained semantics for intensional propositional logics. In: Giordani, A., Malinowski, J. (eds.) Logic in High Definition. Trends in Logical Semantics, Trends in Logic, vol. 56, pp. 13-30. Springer (2021)

[5] Jarmużek, T., Kaczkowski, B.: On some logic with a relation imposed on formulae. Tableau system $\mathcal{F}$. Bull. Sect. Logic 43(1/2), 53-72 (2014)

[6] Jarmużek, T., Klonowski, M.: On logics of strictly-deontic modalities. A semantic and tableau approach. Logic Log. Philos. 29(3), 335-380 (2020)

[7] Jarmużek, T., Klonowski, M.: Some intensional logics defined byrelating semantics and tableau systems. In: Giordani, A., Malinowski, J. (eds.) Logic in high definition. Trends in logical semantics, Trends in Logic, vol. 56, pp. 31-48, Springer (2021)

[8] Jarmużek, T., Malinowski, J.: Boolean connexive logics. Semantic and Tableau approach. Logic Log. Philos. 28(3), 427-448 (2019)

[9] Jarmużek, T., Malinowski, J.: Modal Boolean connexive logics. Semantic and Tableau approach. Bull. Sect. Logic 48(3), 213-243 (2019)

[10] Kapsner, A.: Strong connexivity. Thought. J. Philos. 1(2), 141-145 (2012)

[11] McCall, S.: A History of Connexivity, In: Gabbay, D.M. et al. (eds.) Logic: A History of its Central Concepts, Handbook of the History of Logic, vol. 11, pp. 415-449. Elsevier (2012)

[12] Malinowski, J., Palczewski, R.:Relating Semantics for Connexive Logic. In: Giordani, A., Malinowski, J. (eds) Logic in High Definition. Trends in Logical Semantics, Trends in Logic, vol. 56, pp. 49-65. Springer (2021)

[13] Mares, E., Paoli, F.: C. I. Lewis, E. J. Nelson, and the modern origins of connexive logic. Organ F 26(3), 405-426 (2019)

[14] Omori, H., Wansing, H.: Connexive logics. An overview and current trends. Logic Log. Philos. 28, 371-387 (2019)

[15] Wansing, H.: Connexive Modal Logic, In R. Schmidt et al. (eds.), Advances in Modal Logic Vol. 5, 367-383, King's College Publications, 2005

[16] Wansing, H.: Connexive logic,. In: Zalta, E.N. (ed.) Stanford encyclopedia of philosophy. https://plato.stanford.edu/entries/logic-connexive/. Accessed 10 Oct 2020

Mateusz Klonowski

Department of Logic

Nicolaus Copernicus University in Torun

Moniuszki 16

87-100 Toruń

Poland

e-mail: mateusz.klonowski@umk.pl

Received: May 18, 2021.

Accepted: October 15, 2021. 